\title{
Accuracy of Waist Circumference Measurement using the WHO versus NIH Protocol in Predicting Visceral Adiposity Using Bioelectrical Impedance Analysis among Overweight and Obese Adult Filipinos in a Tertiary Hospital
}

\author{
Leslie Daphne Kawaji ${ }^{1}$ and Joy Arabelle Fontanilla ${ }^{1,2}$
}

${ }^{1}$ Center for Diabetes, Thyroid and Endocrine Disorders, St. Luke's Medical Center, Global City, Taguig, Philippines ${ }^{2}$ Center for Weight Intervention and Nutrition Services, St. Luke's Medical Center, Global City, Taguig, Philippines

\begin{abstract}
Objectives. The study aimed to compare the performance of weight circumference (WC) measurement using the World Health Organization (WHO) versus National Institutes of Health (NIH) protocol in identifying visceral adiposity, and to determine the association of WC with cardiometabolic risk factors among overweight and obese adult Filipinos.

Methodology. A retrospective study involving 221 subjects (99 males, 122 females) evaluated at an outpatient weight intervention center of a tertiary hospital. The WC was measured at the superior border of the iliac crest (WC-NIH) and midway between the lowest rib and the iliac crest (WC-WHO) for each patient. Using visceral fat rating (VF) derived via bioelectrical impedance analysis (BIA) as reference standard, diagnostic accuracy tests for both protocols (using cut-offs of $\geq 90 \mathrm{~cm}$ in males and $\geq 80 \mathrm{~cm}$ in females) were done. Cardiometabolic parameters were also obtained, and binary logistic regression was performed to determine associations with WC.

Results. Among males, WC-WHO had 96\% sensitivity (95\% Cl 88.8\%-99.2\%) and 25\% specificity (95\% Cl 9.77\%-46.7\%) while WC-NIH had $94.7 \%$ sensitivity $(95 \% \mathrm{Cl} 86.9 \%-98.5 \%)$ and $29.2 \%$ specificity $(95 \% \mathrm{Cl} 12.6 \%-51.1 \%)$ to predict high VF $>12$. Among females, WC-WHO had $100 \%$ sensitivity $(95 \% \mathrm{Cl} 90 \%-100 \%)$ and $24.1 \%$ specificity $(95 \% \mathrm{Cl} 15.6 \%-$ $34.5 \%$ ) while WC-NIH had $100 \%$ sensitivity (95\% Cl $90 \%-100 \%)$ and $4.6 \%$ specificity $(95 \% \mathrm{Cl} 1.3 \%-11.4 \%)$. Prevalence of high VF was significantly greater among males - 75.8\% (95\% Cl 66.1\%-83.8\%) vs. 28.7\% (95\% Cl $20.9 \%-37.6 \%)$ in females $(p<0.001)$. Among females, WC-NIH tended to have higher measurements than WC-WHO by an average of $4.67 \mathrm{~cm}$. Females with WC-WHO measurements of at least $80 \mathrm{~cm}$ were approximately four times more likely to have low $(<50 \mathrm{mg} / \mathrm{dL}$ ) HDL levels (cOR 3.82, $p=<0.05$ ), even after adjusting for age (aOR $3.83, p=<0.05)$.

Conclusion. WC measurement using the WHO and NIH protocols were both highly sensitive but had low specificity in predicting high VF estimated via BIA among overweight and obese adult Filipinos in this study. WC-NIH measurements tended to be higher among the females, which may affect classification of central obesity when using this protocol. WC $\geq 80 \mathrm{~cm}$ measured using the WHO protocol was associated with low HDL levels among female subjects. Prospective studies conducted among the general Filipino population are recommended to verify these findings.
\end{abstract}

Key words: waist circumference, central obesity, visceral adiposity

\section{BACKGROUND}

Obesity is a complex condition increasingly recognized as an important risk factor in the development of cardiovascular disease and diabetes mellitus. In the Philippines, the prevalence of overweight and obesity among adults is $37.2 \%{ }^{1}$ Although body mass index (BMI) is most commonly used to classify obesity, it cannot obtain a measurement of fat distribution, particularly central or visceral, which has been associated with increased cardiometabolic risk. ${ }^{2}$ Central obesity is characterized by increased intraabdominal adipose tissue, which has been linked with altered lipoprotein metabolism, ${ }^{3}$ promotion of insulin resistance, ${ }^{4}$ and production of inflammatory adipokines. ${ }^{5}$ In Asians, accumulation of intraabdominal

ISSN 0857-1074 (Print) I eISSN 2308-118x (Online)

Printed in the Philippines

Copyright $(\subset) 2021$ by Kawaji et al.

Received: March 12, 2021. Accepted: August 9, 2021

Published online first: September 2, 2021.

https://doi.org/10.15605/jafes.036.02.13 fat can occur without overt increase in overall body mass. A study by Pagsisihan et al., among rural Filipino subjects noted the occurrence of cardiometabolic diseases at lower BMI cut-offs of $24 \mathrm{~kg} / \mathrm{m}^{2}$ and $23 \mathrm{~kg} / \mathrm{m}^{2}$ in males and females, respectively. ${ }^{6}$

While computed tomography (CT) and magnetic resonance imaging (MRI) are currently the gold standards for the quantification of visceral adiposity, they are considered too expensive, cumbersome and/or invasive for routine clinical use. ${ }^{7}$ Waist circumference (WC) is a reliable surrogate marker of visceral fat mass, and its measurement is recommended in evaluating patients for obesity-related disease risk. Large population studies ${ }^{8-10}$ have shown a significant association between WC and the risk of developing type 2 diabetes 
mellitus (DM) and coronary heart disease independent of other risk factors such as hypertension, blood glucose elevation and dyslipidemia. The relationship between WC and health outcomes persists across different age groups, in males and females, and among several ethnic groups. ${ }^{2}$

Different clinical studies on cardiovascular morbidity and mortality have used different WC measurement sites such as above the iliac crest (recommended by the National Institutes of Health $^{11}$ ), midway between the lowest rib and iliac crest (recommended by the World Health Organization ${ }^{12}$ ), the narrowest portion of the waist or below the lowest rib. In general, it is recommended to use bony anatomic landmarks to serve as easily identifiable fixed guides for measurement. ${ }^{2}$ While there is no universal protocol for WC measurement, several crosssectional studies have attempted to determine which WC measurement site better reflects visceral adiposity and cardiometabolic outcomes in the different ethnic groups.

Bosy-Westphal et al., conducted a study among Caucasian adults and children comparing 3 WC measurement sites, and concluded that WC is better correlated to subcutaneous (SFA) rather than visceral fat areas (VFA). ${ }^{13}$ They determined that WC measured below the lowest rib was the better index of VFA and cardiometabolic risk. Another study in Ireland had similar findings, with WC measured below the lowest rib showing the strongest associations with hypertension, dyslipidemia and DM in both genders. ${ }^{14}$

In a study by $\mathrm{Ma}$ et al., conducted in Taiwan, WC measurement at the iliac crest (WC-IC) and midway between the lowest rib and iliac crest (WC-mid) were compared - they found greater correlations between WCmid and VFA, blood pressure, blood glucose, hemoglobin A1c and lipid levels. ${ }^{15}$ Identification of central obesity using WC-mid was also able to predict the development of DM after 31 months.

A systematic review of 120 studies evaluating different WC measurement criteria showed that the different sites had no impact on morbidity and mortality. ${ }^{16}$ However, these studies determined that the site of WC measurement plays an important role in the evaluation of central obesity and cardiometabolic status. Hence, it may be important to determine which WC measurement site is better used for Filipino subjects in order to identify those at risk for adiposity-related complications and institute timely prevention.

\section{OBJECTIVES}

The general objective of the study was to compare the performance of WC measurement using the WHO versus the NIH protocol in predicting high visceral fat estimated by bioelectrical impedance analysis among overweight and obese adult Filipino patients at St. Luke's Medical Center, Global City.

Specific objectives were to: (1) Compare the sensitivity, specificity, predictive values, likelihood ratios and accuracy of the WHO and NIH protocols; (2) Determine the statistical agreement between the WHO and NIH protocols, disaggregating for males and females; and (3) Compare the association of WC measurements using the WHO versus NIH protocols with the clinical and biochemical cardiometabolic risk factors present among the subjects.

\section{METHODOLOGY}

\section{Study Design}

This was a single-center retrospective study conducted on Filipino patients aged 19 years old and above who were enrolled and evaluated at the Center for Weight Intervention and Nutrition Services (WINS) of St. Luke's Medical Center, Global City from January 2017 to December 2018.

\section{Study Population}

All overweight and obese Filipino patients aged 19 years old and above who were enrolled and evaluated at WINS from January 2017 to December 2018 and who did not fulfill the exclusion criteria were included in this study. Patients with the following conditions that could have interfered with accurate waist circumference measurement were excluded from the study population: abdominal mass, abdominal hernia, abdominal surgery in the past 3 months, and bariatric surgery in the past 3 months. Subjects found to have incomplete data were also excluded from analysis.

\section{Study Procedures}

Patient data which include charts, logbooks and electronic medical records were reviewed by the primary investigator. Demographic data such as age and sex were included.

Anthropometric data obtained were the height, weight, WC and visceral fat rating. Waist circumference expressed in centimeters $(\mathrm{cm})$ was measured using a flexible, nonstretchable plastic tape measure at two sites for every patient: 1. At the horizontal plane on the superior border of the iliac crest (WC-NIH); and 2. At the horizontal plane midway between the lowest rib and the iliac crest (WCWHO). Measurement to the nearest $0.5 \mathrm{~cm}$ was done at the end of a normal expiration and done twice for each site. The higher value was recorded in the event of a discrepancy between the two determinations. All anthropometric measurements were carried out by either of 2 clinical dietitians at WINS.

Visceral fat rating (VF) was obtained after the patient has fasted for at least 2 hours, using a multifrequency segmental body composition analyzer (TANITA MC980MA PLUS) which uses bioelectrical impedance technology. Bioelectrical impedance analysis (BIA) is a practical, rapid and radiation-free modality widely used to analyze body composition. BIA measures the electric resistance between fat and components of other organs, and is used in clinical and epidemiological settings to estimate regional fat distribution. ${ }^{17}$

Studies comparing the accuracy of BIA in determining visceral fat accumulation have been done among Chinese, ${ }^{18}$ Korean $^{19}$ and Japanese $\mathrm{e}^{17,20,21}$ subjects showing moderate to high correlation ( $\mathrm{r}$ 0.605-0.904) with abdominal imaging using CT and MRI. However, there were observed differences in correlation attributed to the sex and $\mathrm{BMI}^{19}$ of the subjects as well as the specification of the BIA machines used (such as single vs multifrequency, ${ }^{18,22}$ bipolar vs tetrapolar electrodes, ${ }^{22}$ or segmental vs whole body $\mathrm{BIA}^{23,24}$ ). Currently, CT and MRI remain the gold standards for quantification of visceral adipose tissue. 
Cardiometabolic risk factors present during the initial evaluation of each patient were included, such as blood pressure (BP), fasting blood sugar (FBS), 2-hour postglucose load values (2-hr OGTT), hemoglobin a1c (HbA1c), lipid profile, as well as the presence of hypertension, diabetes, dyslipidemia and use of relevant medications.

\section{Operational definitions}

Body mass index (BMI) was defined as the subject's weight in kilograms $(\mathrm{kg})$ divided by the height in meters squared $\left(\mathrm{m}^{2}\right)$. Using the WHO criteria for Asians, ${ }^{12}$ the subject is classified as overweight if the BMI is $23.0-24.9 \mathrm{~kg} / \mathrm{m}^{2}$ and obese if the BMI is $\geq 25.0 \mathrm{~kg} / \mathrm{m}^{2}$.

Central obesity was defined as WC of $\geq 90 \mathrm{~cm}$ in males and $\geq 80 \mathrm{~cm}$ in females according to $\mathrm{WHO}$ recommendations. ${ }^{12}$

High VF was defined as visceral fat rating above 12 units, while acceptable level ranges from 1-12 using BIA (TANITA MC 980MA). Visceral fat rating (presented as a value ranging from 1-59) is derived by applying predictive equations to the segmental impedance measurements. ${ }^{22}$ An algorithm was developed by the manufacturer to assign VF values that were based on abdominal imaging by MRI, showing good correlation (r 0.84-0.886). ${ }^{25-27}$

Hypertension (HTN) was defined by systolic blood pressure (SBP) of $\geq 140 \mathrm{mmHg}$ and/or diastolic blood pressure (DBP) of $\geq 90 \mathrm{mmHg}$ on repeated examination, ${ }^{28}$ intake of anti-hypertensive medications, or prior diagnosis by a physician.

Diabetes mellitus (DM) criteria include use of oral hypoglycemic agents and/or insulin, a prior diagnosis by a physician, or if any of the following criteria were present: fasting blood sugar (FBS) $\geq 126 \mathrm{mg} / \mathrm{dL}$, or hemoglobin A1c $(\mathrm{HbA1c}) \geq 6.5 \%$, or 2-hour oral glucose tolerance test (OGTT) result $\geq 200 \mathrm{mg} / \mathrm{dL}$ using $75 \mathrm{~g}$ anhydrous glucose load, or random blood sugar (RBS) $\geq 200 \mathrm{mg} / \mathrm{dL}$ accompanied by signs and symptoms of hyperglycemia such as polydipsia, polyuria and polyphagia. ${ }^{29}$

Diagnosis of dyslipidemia was based on intake of lipidmodifying drugs (such as statins, fibrates etc.), prior diagnosis by a physician, or presence of any of the following abnormal lipid profile results: total cholesterol (TC) $\geq 200 \mathrm{mg} / \mathrm{dL}$, triglyceride (TG) $\geq 150 \mathrm{mg} / \mathrm{dL}$, low density lipoprotein (LDL) $\geq 100 \mathrm{mg} / \mathrm{dL}$, high density lipoprotein (HDL) $<40 \mathrm{mg} / \mathrm{dL}$ in males and $<50 \mathrm{mg} / \mathrm{dL}$ in females. ${ }^{30}$

\section{Sample size}

A minimum of 174 adults satisfying the inclusion/exclusion criteria is required, to compare, with $10 \%$ precision at $5 \%$ level of significance, the performance of WC measurements based on the NIH and WHO protocols in assessing central obesity among overweight and obese adult Filipino patients based on the assumptions that sensitivity and specificity of abdominal circumference are $76 \%$ and $79 \%$, respectively, ${ }^{15}$ and prevalence of central obesity among overweight and obese patients is $63.3 \% .^{31}$

\section{Statistical analysis}

Descriptive statistics was used to summarize the general and clinical characteristics of the participants. Frequency and proportion were used for categorical variables. Shapiro-
Wilk test was used to determine the normality distribution of continuous variables. Continuous quantitative data that met the normality assumption was summarized using mean and standard deviation (SD), while those that do not was described using median and range.

Continuous variables which were normally distributed were compared using the independent t-test. Otherwise, the non-parametric Mann-Whitney U test was used. For categorical variables, Chi-square test was used to compare the outcomes. If the expected percentages in the cells are less than 5\%, Fisher's Exact test was used instead.

Sensitivity, specificity, predictive values, and likelihood ratios of $\mathrm{WC}-\mathrm{WHO}$ and $\mathrm{WC}-\mathrm{NIH}$ (using accepted cutoffs $\geq 90 \mathrm{~cm}$ in males and $\geq 80 \mathrm{~cm}$ in females ${ }^{12,32}$ ) to predict high VF (>12 via BIA) were calculated.

Bland-Altman analysis was used to determine the limits of agreement and the mean difference between WC measurements using the NIH and WHO protocols. Crude and age-adjusted odds ratios with 95\% confidence intervals from binary logistic regression were computed to determine the association between WC and cardiometabolic parameters.

All valid data were included in the analysis. Missing data were neither replaced nor estimated. Null hypothesis was rejected at $0.05 \alpha$-level of significance. STATA 15.0 was used for data analysis.

\section{Ethical considerations}

The protocol of this study adhered to the ethical considerations and ethical principles set out in relevant guidelines, including the Declaration of Helsinki, WHO guidelines, International Conference on HarmonizationGood Clinical Practice, and National Ethics Guidelines for Health Research. The investigators have completed the Good Clinical Practice (GCP) training on the responsible conduct of research with human data. The study only commenced upon the approval of the St. Luke's Medical Center Institutional Ethics Review Committee.

Identifiable data were purged from the study records and each patient was assigned a code number. A master list containing the code number and subject's hospital PIN was kept separately from the research data. The master list and all research data were stored on a password-protected computer which only the investigators could access. These records will be kept for a minimum of 5 years following completion of the study, after which electronic data will be deleted and any existing hard copies will be shredded.

\section{RESULTS}

We enrolled a total of 221 adult Filipinos who were either overweight or obese. They had a median age of 38 years, ranging from 19 to 71 years old. The median BMI was 35.17 $\mathrm{kg} / \mathrm{m}^{2}$ for males and $31.59 \mathrm{~kg} / \mathrm{m}^{2}$ for females. Median WC$\mathrm{NIH}$ measurement was $115.2 \mathrm{~cm}$ for males and $107 \mathrm{~cm}$ for females. Median WC-WHO measurement was $111.5 \mathrm{~cm}$ for males and $99 \mathrm{~cm}$ for females. The median VF was 17 units for males and 10 units for females. Average values for the blood pressure, metabolic parameters, and co-morbidities are enumerated in Table 1. 
Table 1. Clinical characteristics of the overweight and obese adult Filipinos $(n=221)$

\begin{tabular}{|c|c|c|c|}
\hline & All $(n=221)$ & Males $(n=99)$ & Females $(n=122)$ \\
\hline Age (years) & $38(19-71)$ & $38(19-71)$ & $38.5(19-71)$ \\
\hline \multicolumn{4}{|l|}{ Anthropometrics } \\
\hline BMI $\left(\mathrm{kg} / \mathrm{m}^{2}\right)$ & $33.17(23.37-92.64)$ & $35.17(24.59-92.64)$ & $31.59(23.37-67.15)$ \\
\hline 23.0-24.9 (\%) & $10(4.52)$ & $2(2.02)$ & $8(6.56)$ \\
\hline$\geq 25.0(\%)$ & $211(95.48)$ & $97(97.98)$ & $114(93.44)$ \\
\hline \multicolumn{4}{|l|}{ Waist circumference $(\mathrm{cm})$} \\
\hline WC-NIH & $111(75-181)$ & $115.2(83-181)$ & $107(75-154)$ \\
\hline WC-WHO & $106(71-199)$ & $111.5(81-199)$ & $99(71-156)$ \\
\hline Visceral fat rating & $12(4-51)$ & $17(6-51)$ & $10(4-33)$ \\
\hline \multicolumn{4}{|l|}{ Blood pressure $(\mathrm{mmHg})$} \\
\hline Systolic BP & $120(90-170)$ & $120(100-170)$ & $120(90-140)$ \\
\hline Diastolic BP & $80(60-120)$ & $80(60-120)$ & $80(60-100)$ \\
\hline \multicolumn{4}{|l|}{ Metabolic parameters } \\
\hline FBS $(\mathrm{mg} / \mathrm{dL})$ & $97(73-296)$ & $100(76-296)$ & $94.5(73-213)$ \\
\hline 2-hr OGTT (mg/dL) & $128.5(92-329)$ & $135.5(97-226)$ & $124(92-329)$ \\
\hline $\mathrm{HbA} 1 \mathrm{c}(\%)$ & $5.8(4.9-12.5)$ & $5.8(5.2-12.5)$ & $5.7(4.9-8.4)$ \\
\hline Total cholesterol (mg/dL) & $185(85.2-348)$ & $182.9(85.2-295)$ & $186.5(124-348)$ \\
\hline Triglycerides (mg/dL) & $126(49-497)$ & $132(55-497)$ & $124(49-295)$ \\
\hline $\mathrm{HDL}(\mathrm{mg} / \mathrm{dL})$ & $46(27-87)$ & $42(27-84)$ & $47.5(28-87)$ \\
\hline $\mathrm{LDL}(\mathrm{mg} / \mathrm{dL})$ & $118(32-249)$ & $117(32-249)$ & $118.5(54-244.5)$ \\
\hline \multicolumn{4}{|l|}{ Comorbidities } \\
\hline Hypertension (\%) & 79 (35.75) & $45(45.45)$ & $34(27.87)$ \\
\hline With HTN medications & 75 (94.94) & $42(93.33)$ & $33(97.06)$ \\
\hline Diabetes mellitus (\%) & $51(23.08)$ & $29(29.29)$ & $22(18.03)$ \\
\hline With DM medications & $44(86.27)$ & $25(86.21)$ & $19(86.36)$ \\
\hline Dyslipidemia (\%) & $149(67.42)$ & $68(68.69)$ & $81(66.39)$ \\
\hline With lipid medications & $63(42.28)$ & $31(45.59)$ & $32(39.51)$ \\
\hline
\end{tabular}

Overall, WC had a high sensitivity and low specificity to detect VF above 12 via BIA across both NIH and WHO protocols for both sexes (Table 2). The prevalence of high visceral fat was at $75.8 \%(95 \%$ CI $66.1 \%$ to $83.8 \%)$ for males and $28.7 \%$ (95\% CI $20.9 \%$ to $37.6 \%$ ) for females. Consequently, positive predictive values were high for males, while negative predictive values were higher in females. Notably, accuracy was the same for males at $78.8 \%$ for both WC-NIH and WC-WHO. For females, the accuracy was at $32.0 \%$ for WC-NIH and $45.9 \%$ for WC-WHO.

Using Bland-Altman plots (Figures 1-3), we identified the agreement in WC measurement using the $\mathrm{NIH}$ protocol with the WHO protocol. To define the limits of agreement, at least $95 \%$ of the data points should lie within $\pm 1.96 \mathrm{SD}$ of the mean difference. Pitman's test of difference in variance was also done concurrently.
On the average, WC-NIH tended to have higher measurements compared to WC-WHO by $2.19 \mathrm{~cm}(2.5 \%)$. Among males, WC-NIH had lower measurements than WC-WHO by an average of $0.85 \mathrm{~cm}(0.39 \%)$. In contrast, WC-NIH tended to have higher measurements than WCWHO by an average of $4.67 \mathrm{~cm}(4.88 \%)$ among the female subjects. There is a statistically significant difference in variance between the NIH and WHO protocols of WC measurement $(p<0.05)$ overall, in males and in females (Tables 3, 3.1).

Using log-transformed values, the mean difference (log $\mathrm{NIH}$ - log WHO) was calculated in the overall study population, then disaggregated by sex (Figures 4-6). There is a statistically significant difference in variance between the WC measurement protocols $(p<0.05)$. The difference in variance was maintained even after classifying by gender (Table 3.2). On linear regression, there is a negative trend between the differences and averages of the WC values

Table 2. Diagnostic performance of WC-NIH and WC-WHO in predicting high visceral fat at recommended cut-off values, by sex

\begin{tabular}{|c|c|c|c|c|c|c|}
\hline & \multicolumn{3}{|c|}{ Males $(n=99)$} & \multicolumn{3}{|c|}{ Females $(n=122)$} \\
\hline & WC-NIH & WC-WHO & $p$ & WC-NIH & WC-WHO & $p$ \\
\hline Prevalence of high visceral fat (\%) & \multicolumn{2}{|c|}{$75.8(66.1-83.8)$} & & \multicolumn{2}{|c|}{$28.7(20.9-37.6)$} & $<0.001^{\mathrm{a}}$ \\
\hline Sensitivity (\%) & $94.7(86.9-98.5)$ & $96(88.8-99.2)$ & & $100(90.0-100)$ & $100(90.0-100)$ & \\
\hline Specificity (\%) & $29.2(12.6-51.1)$ & $25(9.77-46.7)$ & & $4.6(1.3-11.4)$ & $24.1(15.6-34.5)$ & \\
\hline PPV (\%) & $80.7(70.9-88.3)$ & $80(70.2-87.7)$ & & $29.7(21.6-38.8)$ & $34.7(25.5-44.8)$ & \\
\hline NPV (\%) & $63.6(30.8-89.1)$ & $66.7(29.9-92.5)$ & & $100(39.8-100)$ & $100(83.9-100)$ & \\
\hline LR+ & $1.34(1.03-1.74)$ & $1.28(1.01-1.62)$ & & $1.05(1.0-1.1)$ & $1.32(1.17-1.48)$ & \\
\hline LR- & $0.18(0.06-0.57)$ & $0.16(0.04-0.59)$ & & 0 & 0 & \\
\hline Accuracy (\%) & $78.8(69.4-86.4)$ & $78.8(69.4-86.4)$ & & $32(23.8-41.0)$ & $45.9(36.9-55.2)$ & \\
\hline $\begin{array}{l}\text { Data are presented as an estimate } \\
\text { Test positive: Females, WC } \geq 80 \mathrm{~cm} \text {; } \\
\text { Disease positive: } V F>12 \\
\text { a, p-value }<0.05 \text { on males vs female }\end{array}$ & $\begin{array}{l}\text { confidence interva } \\
\mathrm{s}, \mathrm{WC} \geq 90 \mathrm{~cm}\end{array}$ & & & & & \\
\hline
\end{tabular}



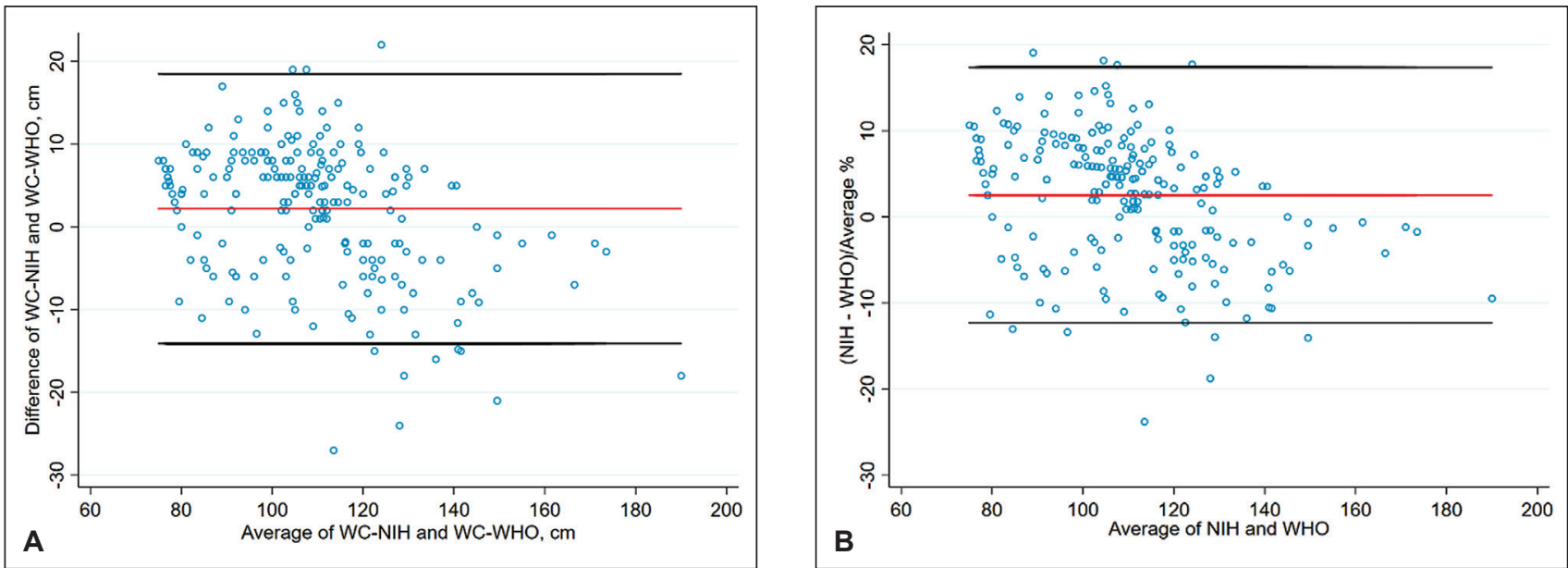

Figure 1. Bland Altman plot depicting agreement between WC-NIH and WC-WHO measurements in the overall study population. (A) Differences presented in $\mathrm{cm}$, with an agreement range from -14.12 to $+18.50 \mathrm{~cm}$; (B) Percentage difference presented, with an agreement range from $-12.33 \%$ to $+17.37 \%$.
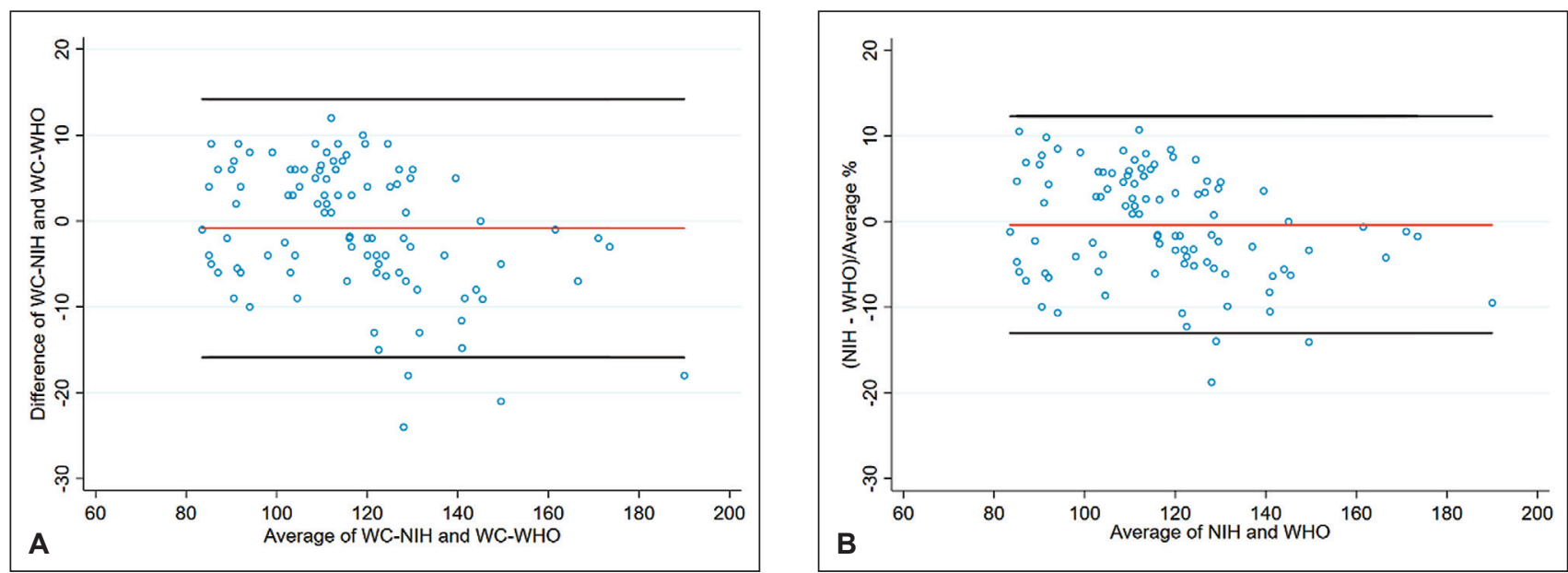

Figure 2. Bland Altman plot depicting agreement between WC-NIH and WC-WHO measurements among the male subjects. (A) Differences presented in $\mathrm{cm}$, with an agreement range from -15.89 to $+14.18 \mathrm{~cm}$; (B) Percentage difference presented, with an agreement range from $-13.04 \%$ to $+12.27 \%$.
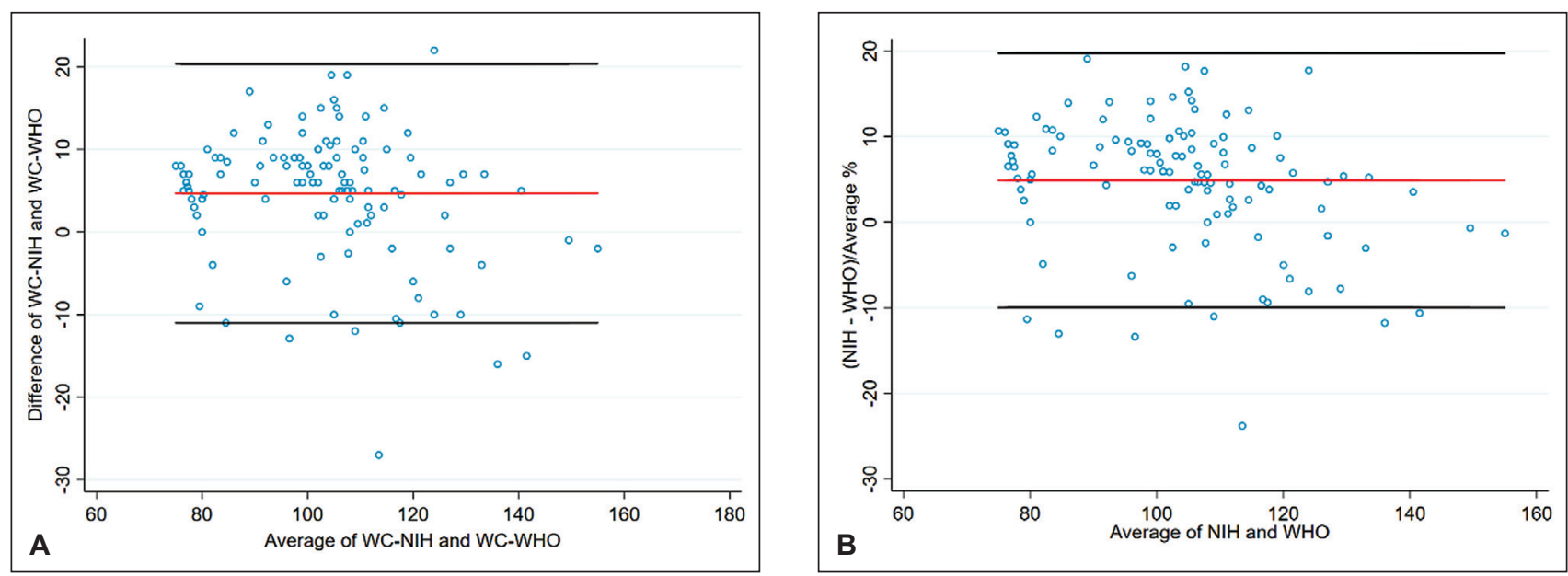

Figure 3. Bland Altman plot depicting agreement between WC-NIH and WC-WHO measurements among the female subjects. (A) Differences presented in $\mathrm{cm}$, with an agreement range from -11.01 to $+20.34 \mathrm{~cm}$; (B) Percentage difference presented, with an agreement range from -9.99 to $+19.75 \%$. 
Table 3. Bland-Altman agreement between WC-NIH and WC-WHO

\begin{tabular}{|c|c|c|c|}
\hline & Overall & Males & Females \\
\hline $\mathrm{N}$ & 221 & 99 & 122 \\
\hline Limits of Agreement & -14.12 to 18.50 & -15.89 to 14.18 & -11.01 to 20.34 \\
\hline Mean difference between WC-NIH and WC-WHO $(95 \% \mathrm{CI}), \mathrm{cm}$ & 2.19 (1.11 to 3.27$)$ & $-0.85(-2.35$ to 0.65$)$ & $4.67(3.26$ to 6.07$)$ \\
\hline Range, $\mathrm{cm}$ & 75.0 to 190.0 & 83.5 to 190.0 & 75.0 to 155.0 \\
\hline Pitman's Test of difference in variance $(r)$ & -0.382 & -0.375 & -0.24 \\
\hline P-Value & $<0.001^{*}$ & $<0.001^{*}$ & $0.008^{*}$ \\
\hline
\end{tabular}

Limits of agreement $=$ mean diff. $\pm 1.96^{*}$ std dev. We expect that $95 \%$ of the differences will lie within the limits of agreement.

${ }^{*} p$-value $<0.05$

Table 3.1. Bland-Altman agreement between WC-NIH and WC-WHO using percentage differences

\begin{tabular}{|c|c|c|c|}
\hline & Overall & Males & Females \\
\hline $\mathrm{N}$ & 221 & 99 & 122 \\
\hline Limits of Agreement, \% & -12.33 to 17.37 & -13.04 to 12.27 & -9.99 to 19.75 \\
\hline Mean difference between WC-NIH and WC-WHO (95\% CI), \% & $2.52(1.52$ to 3.52$)$ & $-0.39(-1.66$ to 0.88$)$ & 4.88 (3.54 to 6.23$)$ \\
\hline Range, cm & 75.0 to 190.0 & 83.5 to 190.0 & 75.0 to 155.0 \\
\hline Pitman's Test of difference in variance ( $r$ ) & -0.38 & -0.305 & -0.301 \\
\hline P-Value & $<0.001^{*}$ & $0.002^{*}$ & $0.001^{*}$ \\
\hline
\end{tabular}

Limits of agreement $=$ mean diff. $\pm 1.96 *$ std dev. We expect that $95 \%$ of the differences will lie within the limits of agreement. ${ }^{*} \mathrm{p}$-value $<0.05$

Table 3.2. Bland-Altman agreement between log-transformed values of WC-NIH and WC-WHO

\begin{tabular}{|c|c|c|c|}
\hline & Overall & Males & Females \\
\hline $\mathrm{N}$ & 221 & 99 & 122 \\
\hline Limits of Agreement & -0.12 to 0.17 & -0.13 to 0.12 & -0.10 to 0.20 \\
\hline Mean difference between WC-NIH and WC-WHO $(95 \% \mathrm{CI})$ & $0.025(0.015$ to 0.035$)$ & $-0.004(-0.017$ to 0.009$)$ & $0.049(0.036$ to 0.062$)$ \\
\hline Range & 4.32 to 5.25 & 4.43 to 5.25 & 4.32 to 5.04 \\
\hline Pitman's Test of difference in variance ( $r$ ) & -0.368 & -0.289 & -0.282 \\
\hline P-Value & $<0.001^{*}$ & $0.004^{*}$ & $0.002^{*}$ \\
\hline
\end{tabular}

Limits of agreement $=$ mean diff. $\pm 1.96^{*}$ std dev. We expect that $95 \%$ of the differences will lie within the limits of agreement.

${ }^{*}$ p-value $<0.05$

using the NIH and WHO protocols $(\mathrm{p}<0.05)$, suggesting the presence of proportional bias. The negative trend was still observed after classifying by gender (Table 3.3).

Females with WC of at least $80 \mathrm{~cm}$ via the WHO protocol were approximately four times more likely to have a low HDL level <50 mg/dL (cOR 3.82, 95\% CI 1.36-10.65).

Table 3.3. Relationship between the mean and difference of waist circumference measurements using WC-NIH and WC-WHO

\begin{tabular}{|c|c|c|}
\hline & Beta Coefficient $(95 \% \mathrm{Cl})$ & $p$ \\
\hline \multicolumn{3}{|c|}{ Average of WC-NIH and WC-WHO } \\
\hline Overall & $-0.15(-0.20$ to -0.10$)$ & $<0.001^{*}$ \\
\hline Males & $-0.10(-0.18$ to -0.03$)$ & $<0.001^{*}$ \\
\hline Females & $-0.13(-0.21$ to -0.05$)$ & $0.002^{*}$ \\
\hline
\end{tabular}

This association was maintained even after adjusting for age (aOR 3.83, 95\% CI 1.37-10.70). We have insufficient evidence to demonstrate an association of WC with the rest of the cardiometabolic parameters in the subjects (Tables 4, 4.1).

\section{DISCUSSION}

To the best of our knowledge, this is the first study comparing WC measurement sites in evaluating central adiposity among Filipino subjects. Using the current recommended cutoffs ( $\geq 90 \mathrm{~cm}$ for males and $\geq 80 \mathrm{~cm}$ for females), sensitivity of both WC measurement sites for identification of elevated VF on BIA was similarly high in both genders. As a screening tool for central obesity, this attribute may be beneficial. However, specificity of both WC measurement sites was poor in both males

Table 4. Association between waist circumference and cardiometabolic factors in overweight and obese Filipino adults $(\mathrm{N}=122)$

\begin{tabular}{|c|c|c|c|c|}
\hline & \multicolumn{2}{|c|}{ Males } & \multicolumn{2}{|c|}{ Females } \\
\hline & WC-NIH & WC-WHO & WC-NIH & WC-WHO \\
\hline $\mathrm{SBP} \geq 130$ & $3.58(0.73-17.54)$ & $6.40(0.77-53.32)$ & $2.33(0.12-44.82)$ & $2.65(0.57-12.24)$ \\
\hline $\mathrm{DBP} \geq 85$ & $11.99(0.68-210.44)$ & $9.58(0.54-170.11)$ & $1.06(0.05-20.80)$ & $6.01(0.34-105.46)$ \\
\hline FBS $\geq 100$ & $1.44(0.41-5.07)$ & $2.50(0.59-10.63)$ & $0.53(0.07-3.92)$ & $1.93(0.65-5.69)$ \\
\hline 2-hr OGTT $\geq 140$ & $3.96(0.18-89.19)$ & $2.60(0.11-62.57)$ & $0.15(0.01-1.74)$ & $1.35(0.06-30.00)$ \\
\hline $\mathrm{HbA} 1 \mathrm{c}>5.6$ & $1.75(0.32-9.50)$ & $1.28(0.26-6.29)$ & $1.38(0.08-23.17)$ & $1.11(0.27-4.60)$ \\
\hline $\mathrm{TC} \geq 200$ & $0.62(0.40-0.18)$ & $0.66(0.16-2.62)$ & $1.92(0.19-18.99)$ & $2.28(0.77-6.70)$ \\
\hline TG $<150$ & $1.22(0.33-4.53)$ & $0.57(0.11-2.92)$ & $0.60(0.20-1.85)$ & $0.56(0.17-1.79)$ \\
\hline Low HDL ( $<40$ males; $<50$ females) & $1.85(0.46-7.44)$ & $5.85(0.70-48.73)$ & $3.81(0.38-37.68)$ & $3.82(1.36-10.65)^{*}$ \\
\hline LDL >100 & $0.82(0.22-3.02)$ & $0.95(0.22-4.07)$ & $1.48(0.15-14.70)$ & $0.75(0.28-2.00)$ \\
\hline
\end{tabular}


Table 4.1. Association between waist circumference and cardiometabolic factors, adjusted for age $(\mathrm{N}=221)$

\begin{tabular}{|c|c|c|c|c|}
\hline & \multicolumn{2}{|c|}{ Males } & \multicolumn{2}{|c|}{ Females } \\
\hline & WC-NIH & WC-WHO & WC-NIH & WC-WHO \\
\hline $\mathrm{SBP} \geq 130 \mathrm{mmHg}$ & $3.82(0.76-19.18)$ & $6.57(0.78-55.18)$ & $2.37(0.12-46.75)$ & $3.31(0.65-16.77)$ \\
\hline $\mathrm{DBP} \geq 85 \mathrm{mmHg}$ & $11.05(0.62-195.53)$ & $8.98(0.51-159.64)$ & $0.98(0.05-19.56)$ & $6.61(0.37-118.94)$ \\
\hline $\mathrm{FBS} \geq 100 \mathrm{mg} / \mathrm{dL}$ & $1.93(0.65-5.69)$ & $4.29(0.83-22.12)$ & $0.53(0.07-4.12)$ & $2.51(0.72-8.71)$ \\
\hline 2-hr OGTT $\geq 140 \mathrm{mg} / \mathrm{dL}$ & $4.18(0.18-99.13)$ & $2.70(0.11-66.24)$ & $0.18(0.01-2.06)$ & $1.20(0.05-29.61)$ \\
\hline $\mathrm{HbA} 1 \mathrm{c}>5.6 \%$ & $1.11(0.27-4.60)$ & $1.53(0.28-8.32)$ & $1.49(0.09-26.17)$ & $1.60(0.35-7.44)$ \\
\hline $\mathrm{TC} \geq 200 \mathrm{mg} / \mathrm{dL}$ & $2.28(0.77-6.70)$ & $0.64(0.16-2.56)$ & $1.98(0.20-19.80)$ & $2.37(0.79-7.12)$ \\
\hline $\mathrm{TG}<150 \mathrm{mg} / \mathrm{dL}$ & $0.56(0.17-1.79)$ & $0.58(0.11-2.97)$ & $0.61(0.20-1.85)$ & $0.56(0.17-1.79)$ \\
\hline Low HDL mg/dL (<40 males; $<50$ females) & $1.52(0.36-6.33)$ & $5.66(0.66-48.20)$ & $3.78(0.38-37.44)$ & $3.83(1.37-10.70)^{*}$ \\
\hline LDL >100 & $0.75(0.28-2.00)$ & $1.09(0.25-4.81)$ & $1.44(0.14-14.34)$ & $0.76(0.28-2.02)$ \\
\hline
\end{tabular}

and females. This may be due to the nature of the study population, which consisted of overweight and obese individuals with almost all subjects exceeding the WC cutoffs. Accuracy of both WC-WHO and WC-NIH was also lower for the female subjects compared to the males, which may be due to the significantly lower prevalence of high VF among the female study population.

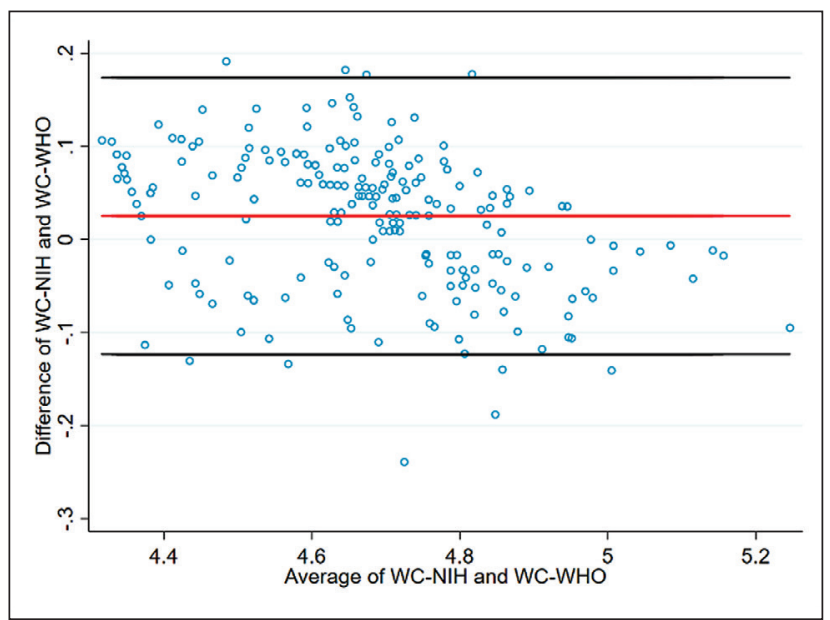

Figure 4. Bland-Altman plot depicting agreement between log-transformed WC-NIH and WC-WHO values in the overall study population. Agreement range was from -0.12 to +0.17 .

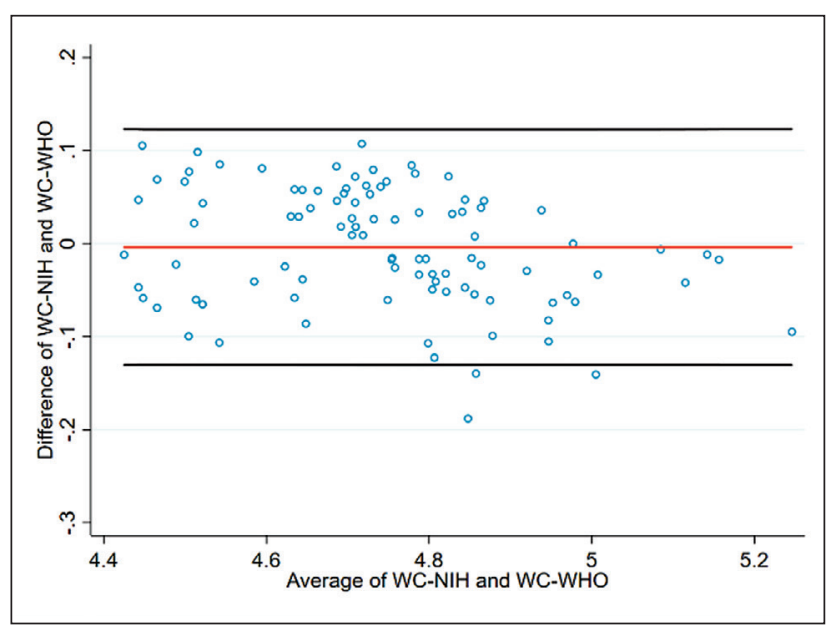

Figure 5. Bland-Altman plot depicting agreement between log-transformed WC-NIH and WC-WHO values among the male subjects. Agreement range was from -0.13 to 0.12 .
In this study, diagnostic parameters of WC-WHO and WC-NIH were similar, with overlapping 95\% confidence intervals of the sensitivity, specificity, predictive values, likelihood ratios, and diagnostic accuracy between both methods. This is in contrast to the findings of $\mathrm{Ma}$ et al., which determined that WC measured according to the WHO protocol was superior in detecting high visceral fat in females. ${ }^{15}$ However, an important difference in their study is that they used CT to quantify VFA.

Based on Bland-Altman analysis, there was no agreement between WC measurements using the WHO and NIH protocols in this study. While we noted statistically significant differences in the variance of both WC measurement protocols (i.e., they are statistically not interchangeable), the average difference of $4.67 \mathrm{~cm}$ between WC-NIH from WC-WHO among the female subjects (compared to only $0.85 \mathrm{~cm}$ in males) may be more clinically significant - likely due to sex differences in body fat distribution. As seen in other similar studies, WC measurement location has a greater impact in females. ${ }^{13,15}$ Classification of central obesity therefore might differ depending on the WC measurement protocol used, particularly among women. This supports the recommendation of the International Atherosclerosis Society (IAS) and International Chair on Cardiometabolic Risk (ICCR) Working Group on Visceral Obesity to be consistent in the use of WC measurement protocols. ${ }^{33}$

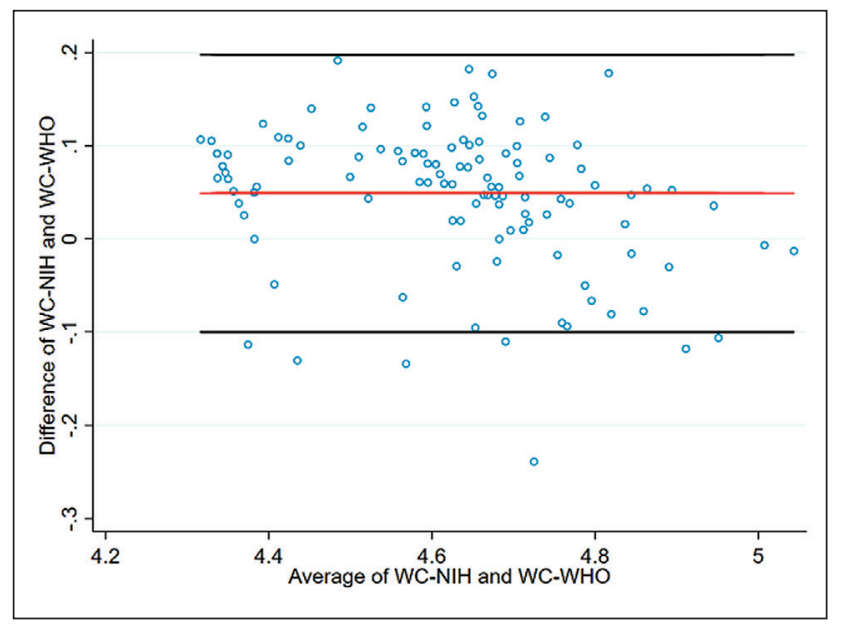

Figure 6. Bland-Altman plot depicting agreement between log-transformed WC-NIH and WC-WHO values among the female subjects. Agreement range was from -0.10 to +0.20 . 
Among the clinical and biochemical cardiometabolic parameters, only low HDL ( $<50 \mathrm{mg} / \mathrm{dL})$ among the females had an association with WC-WHO even after adjusting for age. In contrast, Ma et al., determined that both WC$\mathrm{NIH}$ and WC-WHO were associated with blood pressure, glucose levels, TG and HDL. ${ }^{15}$

Our study compared the relationships of WC-WHO and WC-NIH with visceral fat and cardiometabolic risk factors. While adequate comparison of parameters was attempted, several limitations are present in the current study. First is that other WC measurement sites were not evaluated. Other studies have concluded that WC measurement using the lowest rib was more reproducible

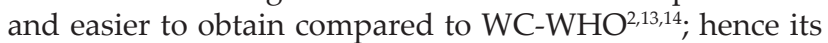
assessment could be clinically relevant.

Second, the gold standard methods (CT or MRI) for quantification of visceral adipose tissue were not utilized. While there are several studies that explored the correlation of BIA with the gold standards for visceral fat quantification, these studies were conducted on a relatively small number of subjects. BIA equations may also be population-specific, ${ }^{22}$ and currently there are no studies evaluating the accuracy of BIA and visceral fat rating conducted among Filipinos.

Third, there were 2 observers who performed WC measurements throughout the study period, although only 1 observer performed both WC measurement methods in each patient, and interobserver variability could be a factor.

Fourth, temporal relationships between WC measurement and cardiometabolic diseases could not be established due to the cross-sectional study design.

Lastly, the single-center study population was composed of overweight or obese subjects hence our findings may not be generalizable to the non-overweight/non-obese Filipino.

\section{CONCLUSION}

Based on this study, WC measurement using the WHO and NIH protocols showed similarly high sensitivity but low specificity in identifying high VF estimated via BIA among overweight and obese adult Filipinos. WC$\mathrm{NIH}$ measurements tended to be higher among the females owing to sex-related body fat distribution, which may affect classification of central obesity when using this protocol.

In this study, female subjects with an elevated WC measured using the WHO protocol were more likely to have low HDL levels, even after adjusting for age. We were unable to demonstrate an association between WC and the rest of the cardiometabolic factors among the subjects.

Prospective studies conducted among the general Filipino population using gold standard methods such as CT or MRI to quantify visceral adiposity are recommended to further determine the most appropriate WC measurement site and cut-offs to define central obesity, and further establish associations with cardiometabolic risk factors.

\section{Acknowledgments}

The authors wish to thank the staff and consultants of the Center for Diabetes, Thyroid and Endocrine Disorders, and the Center for Weight Intervention and Nutrition Services of St. Luke's Medical Center, Global City for their help and guidance in the preparation of the study.

\section{Statement of Authorship}

Both authors certified fulfillment of ICMJE authorship criteria.

\section{Author Disclosure}

Both authors declared no conflicts of interest.

\section{Funding Source}

None.

\section{References}

1. Food and Nutrition Research Institute - Expanded National Nutritional Survey 2018. https://www.fnri.dost.gov.ph/images/ sources/eNNS2018/AdultsandElderly.pdf.

2. Klein S, Allison DB, Heymsfield SB, et al. Waist circumference and cardiometabolic risk: A consensus statement from Shaping America's Health: Association for Weight Management and Obesity Prevention; NAASO, The Obesity Society; the American Society for Nutrition; and the American Diabetes Association. Am J Clinical Nutr. 2007;85(5):1197-1202. PMID: 17490953. https://doi.org/10.1093/ ajcn/85.5.1197.

3. Sinha R, Dufour S, Petersen KF, et al. Assessment of skeletal muscle triglyceride content by (1)H nuclear magnetic resonance spectroscopy in lean and obese adolescents: relationships to insulin sensitivity, total body fat, and central adiposity. Diabetes. 2002;51(4):1022-7. PMID: 11916921. https://doi.org/10.2337/diabetes.51.4.1022.

4. Seppälä-Lindroos A, Vehkavaara S, Häkkinen AM, et al. Fat accumulation in the liver is associated with defects in insulin suppression of glucose production and serum free fatty acids independent of obesity in normal men. J Clin Endocrinol Metab. 2002;87(7):3023-28. PMID: 12107194. https://doi.org/10.1210/jcem. 87.7.8638.

5. Björntorp P. Body fat distribution, insulin resistance, and metabolic diseases. Nutrition. 1997;13(9):795-803. PMID: 9290093. https://doi. org/10.1016/s0899-9007(97)00191-3.

6. Pagsisihan D, Sandoval MA, Paz-Pacheco E, Jimeno C. Low indices of overweight and obesity are associated with cardiometabolic diseases among adult Filipinos in a rural community. J ASEAN Fed Endocr Soc. 2016;31(2): 97-105. https://doi.org/10.15605/jafes.031.02.04.

7. Shuster A, Patlas M, Pinthus JH, Mourtzakis M. The clinical importance of visceral adiposity: A critical review of methods for visceral adipose tissue analysis. Br J Radiol. 2012;85(1009):1-10. PMID: 21937614. PMCID: PMC3473928. https://doi.org/10.1259/bjr/38447238.

8. Yusuf S, Hawken S, Ounpuu S, et al. Obesity and the risk of myocardial infarction in 27,000 participants from 52 countries: A case-control study. Lancet. 2005;366(9497):1640-49. PMID: 16271645. https:// doi.org/10.1016/S0140-6736(05)67663-5.

9. Suk SH, Sacco RL, Boden-Albala B, et al. Abdominal obesity and risk of ischemic stroke: The Northern Manhattan Stroke Study. Stroke. 2003;34(7):1586-92. PMID: 12775882. https://doi.org/10.1161/01.STR. $0000075294.98582 .2 \mathrm{~F}$.

10. Visscher TL, Seidell JC, Molarius A, van der Kuip D, Hofman A, Witteman JC. A comparison of body mass index, waist-hip ratio and waist circumference as predictors of all-cause mortality among the elderly: The Rotterdam study. Int J Obes Relat Metab Disord. 2001;25(11):1730-5. PMID: 11753597. https://doi.org/10.1038/ sj.ijo.0801787.

11. Grundy SM, Cleeman JI, Daniels SR, et al. Diagnosis and management of the metabolic syndrome: An American Heart Association/Nationa Heart, Lung, and Blood Institute Scientific Statement. Circulation. 2005;112(17):2735-52. PMID: 16157765. https://doi.org/10.1161/ CIRCULATIONAHA.105.169404.

12. World Health Organization. Regional Office for the Western Pacific The Asia-Pacific perspective: Redefining obesity and its treatment. Sydney: Health Communications Australia; 2000. https://apps. who.int/iris/bitstream/handle/10665/206936/0957708211_eng. pdf? sequence $=1$ \&isAllowed $=y$.

13. Bosy-Westphal A, Booke C-A, Blocker T, et al. Measurement site for waist circumference affects its accuracy as an index of visceral and abdominal subcutaneous fat in a Caucasian population. Nutr. 2010;140(5):956-61. PMID: 20335625. https://doi.org/10.3945/ jn.109.118737.

14. Millar SR, Perry IJ, Van den Broeck J, Phillips CM. Optimal central obesity measurement site for assessing cardiometabolic and type 2 diabetes risk in middle-aged adults. PLoS One. 2015;10(6):e0129088. 
PMID: 26042771. PMCID: PMC4456242. https://doi.org/10.1371/ journal.pone.0129088.

15. Ma WY, Yang CY, Shih SR, et al. Measurement of waist circumference: Midabdominal or iliac crest? Diabetes Care. 2013;36(6):1660-6. PMID: 23275359. PMCID: PMC3661855. https://doi.org/10.2337/dc12-1452.

16. Ross R, Berentzen T, Bradshaw AJ, et al. Does the relationship between waist circumference, morbidity and mortality depend on measurement protocol for waist circumference? Obesity Rev. 2008;9(4):312-25. PMID: 17956544. https://doi.org/10.1111/j.1467-789X.2007.00411.x.

17. Nagai M, Komiya H, Mori Y, Ohta T, Kasahara Y, Ikeda Y. Estimating visceral fat area by multifrequency bioelectrical impedance. Diabetes Care. 2010;33(5):1077-9. PMID: 20150289. PMCID: PMC2858179. https://doi.org/10.2337/dc09-1099.

18. Wang JG, Zhang Y, Chen HE, et al. Comparison of two bioelectrical impedance analysis devices with dual energy $\mathrm{x}$-ray absorptiometry and magnetic resonance imaging in the estimation of body composition. J Strength Cond Res. 2013;27(1):236-43. PMID: 22344056. https://doi.org/10.1519//SC.0b013e31824f2040.

19. Lee DH, Park KS, Ahn S, et al. Comparison of abdominal visceral adipose tissue area measured by computed tomography with that estimated by bioelectrical impedance analysis method in Korean subjects. Nutrients. 2015;7(12):10513-24. PMID: 26694460. PMCID: PMC4690100. https://doi.org/10.3390/nu7125548.

20. Shoji K, Maeda K, Nakamura T, Funahashi T, Matsuzawa $\mathrm{Y}$, Shimomura I. Measurement of visceral fat by abdominal bioelectrical impedance analysis is beneficial in medical checkup. Obes Res Clin Pract. 2008;2(4):I-II. PMID: 24351854. https://doi.org/10.1016/i. orcp.2008.09.001

21. Ryo M, Maeda K, Onda T, et al. A new simple method for the measurement of visceral fat accumulation by bioelectrical impedance Diabetes Care. 2005;28(2):451-3. PMID: 15677816. https://doi org/10.2337/diacare.28.2.451

22. Bosy-Westphal A, Later W, Hitze B, et al. Accuracy of bioelectrical impedance consumer devices for measurement of body composition in comparison to whole body magnetic resonance imaging and dual X-ray absorptiometry. Obes Facts. 2008;1(6):319-24. PMID: 20054195. PMCID: PMC6452160. https://doi.org/10.1159/000176061

23. Park KS, Lee DH, Lee JE, et al. Comparison between two methods of bioelectrical impedance analyses for accuracy in measuring abdominal visceral fat area. J Diabetes Complications. 2016;30(2): 343-9. PMID: 26620129. https://doi.org/10.1016/j.jdiacomp.2015.10.014.

24. Lu HK, Chen YY, Yeh C, et al. Discrepancies between leg-to-leg bioelectrical impedance analysis and computerized tomography in abdominal visceral fat measurement. Sci Rep. 2017;7(1):9102. PMID 28831095. PMCID: PMC5567333. htts://doi.org/10.1038/s41598-01708991-y.
25. Tanita Institute Contract Study. Algorithm development for estimating visceral fat rating. SB Heymsfield MD. Columbia University College of Physicians and Surgeons; 2004.

26. Wang Z. Japanese-American differences in visceral adiposity and a simplified estimation method for visceral adipose tissue. North American Association for the Study of Obesity Annual Meeting. Abstract 518-P; 2004

27. Tanita Corporation. Tanita Technical Bulletin: Visceral fat measurement. https://www.tanita.com/data/File/AdditionalResearch/ VisceralFatMeasurmentp1.pdf?rev=72DE.

28. Unger T, Borghi C, Charchar F, et al. 2020 International Society of Hypertension Global Hypertension Practice Guidelines. Hypertension. 2020;75(6):1334-57. PMID: 32370572. https://doi.org/ 10.1161/HYPERTENSIONAHA.120.15026.

29. American Diabetes Association. Classification and Diagnosis of Diabetes: Standards of Medical Care in Diabetes - 2021. Diabetes Care. 2021;44 (Suppl 1):S15-33. PMID: 33298413. https://doi.org/10.2337/ dc21-S002.

30. National Cholesterol Education Program (NCEP) Expert Panel on Detection, Evaluation, and Treatment of High Blood Cholesterol in Adults (Adult Treatment Panel III). Third report of the National Cholesterol Education Program (NCEP) expert panel on detection, evaluation, and treatment of high blood cholesterol in adults (Adult Treatment Panel III) final report. Circulation. 2002;106(25): 3143-421. PMID: 12485966.

31. Hu L, Huang X, You C, et al. Prevalence of overweight, obesity, abdominal obesity and obesity-related risk factors in southern China. PLoS One. 2017;12(9):e0183934. PMID: 28910301. PMCID: PMC5598943. https://doi.org/10.1371/journal.pone.0183934.

32. Alberti KG, Eckel RH, Grundy SM, et al. Harmonizing the metabolic syndrome: A joint interim statement of the International Diabetes Federation Task Force on Epidemiology and Prevention; National Heart, Lung, and Blood Institute; American Heart Association; World Heart Federation; International Atherosclerosis Society; and International Association for the Study of Obesity. Circulation. 2009;120(16):1640-5. PMID: 19805654. https://doi.org/ 10.1161/CIRCULATIONAHA.109.192644.

33. Ross R, Neeland IJ, Yamashita S, et al. Waist circumference as a vital sign in clinical practice: A consensus statement from the IAS and ICCR Working Group on Visceral Obesity. Nat Rev Endocrinol. 2020;16(3):177-89. PMID: 32020062. PMCID: PMC7027970. https:// doi.org/10.1038/s41574-019-0310-7.

Authors are required to accomplish, sign and submit scanned copies of the JAFES Author Form consisting of: (1) Authorship Certification, that authors contributed substantially to the work, that the manuscript has been read and approved by all authors, and that the requirements for authorship have been met by each author; (2) the Author Declaration, that the article represents original material that is not being considered for publication or has not been published or accepted for publication elsewhere, that the article does not infringe or violate any copyrights or intellectual property rights, and that no references have been made to predatory/suspected predatory journals; (3) the Author Contribution Disclosure, which lists the specific contributions of authors; (4) the Author Publishing Agreement which retain author copvright, grants publishing and distribution rights to JAFES, and allows JAFES to apply and enforce an Attribution-Non-Commercial Creative Commons user license; and (5) the Conversion to Visual Abstracts ( ${ }^{\circ}$ optional for original articles only) to improve dissemination to practitioners and lay readers Authors are also required to accomplish, sign, and submit the signed ICMJE form for Disclosure of Potential Conflicts of Interest. For original articles, authors are required to submit a scanned copy of the Ethics Review Approval of their research as well as registration in trial registries as appropriate. For manuscripts reporting data from studies involving animals, authors are required to submit a scanned copy of the Institutional Animal Care and Use Committee approval. For Case Reports or Series, and Images in Endocrinology, consent forms, are required for the publication of information about patients; otherwise, appropriate ethical clearance has been obtained from the institutional review board. Articles and any other material published in the JAFES represent the work of the author(s) and should not be construed to reflect the opinions of the Editors or the Publisher. 